\title{
Developmental Social Robotics: An Applied Perspective
}

\author{
Amit Kumar Pandey ${ }^{1}$ - Rachid Alami ${ }^{2,3}$ - Kazuhiko Kawamura ${ }^{4}$
}

Published online: 8 July 2015

(c) Springer Science+Business Media Dordrecht 2015

For robots to coexist with us in harmony and be our companion, they should be able to explicitly reason about humans, their presence, the social and human-centered environment, and the social-cultural norms, to behave in socially expected and accepted manner. To develop such capabilities, from psychology, child developmental and human behavioral research, we can identify some of the key ingredients, such as the abilities to distinguish between self and others, and to reason about affordance, perspective taking, shared spaces, social signals, emotions, theory of mind, social situation, etc., and the capability to develop social intelligence through the process of social learning. Researchers across the world are working to equip robots with some of these aspects from diverse perspectives and developing various interesting and innovative applications. This special issue is intended to reflect some of those high-quality research works, results and potential applications.

From the point of view of understanding the fundamentals of developmental process of children, interesting aspects

Amit Kumar Pandey

akpandey@aldebaran.com

Rachid Alami

rachid.alami@laas.fr

Kazuhiko Kawamura

kaz.kawamura@vanderbilt.edu

1 A-Lab, Aldebaran SoftBank Group, Paris, France

2 CNRS, LAAS, 7 avenue du colonel Roche, 31400 Toulouse, France

3 Univ de Toulouse, LAAS, 31400 Toulouse, France

4 Department of Electrical Engineering and Computer Science, Vanderbilt University, Nashville, USA can be observed. Jean Piaget and Bärbel Inhelder observe it as a series of stages developed over time. Jerome Seymour Bruner, Rose R. Olver and Patricia M. Greenfield see it as more continuous process that can even be speeded-up. Lev S. Vygotsky sees development to be strongly coupled with social and cultural context. The aim of this special issue is not to contribute in the understanding of fundamental process of social development. Our focus is to identify and address some of the important milestones, steps, and building blocks of development of social intelligence, and how to adapt, extend and apply them into robotics by identifying as well as addressing the underlying $R \& D$ challenges when combination of such blocks should be functioning together.

The special issue consists of ten high quality research papers along three broader categories:

(i) Methodologies for embodiment of some basic social developmental blocks (papers 1-3).

(ii) User perception, evaluation and design guidelines for social embodiment of robots with some basic social developmental blocks (papers 4-7).

(iii) Potential societal applications of social robots embodied with some of the social developmental blocks (papers $8-10)$.

Some of these papers are significantly enriched versions of the papers accepted in the proceedings of International Workshop on Developmental Social Robotics (DevSoR): Reasoning about Human, Perspective, Affordances and Effort for Socially Situated Robots, IEEE/RSJ International Conference on Intelligent Robots and Systems (IROS) 2013, 7 Nov 2013 Tokyo (Japan). Following section will highlight the papers constituting the special issue. 


\section{Overview of the Papers}

The first paper titles "The role of functional affordances in socializing robots", authored by Iman Awaad, Gerhard K. Kraetzschmar and Joachim Hertzberg, provides mechanism to embody the notion and usability of functional affordances of objects by modelling them in description logics. The idea is to use the functional affordances in finding or substituting the right or most suitable available object for achieving a goal. Affordance being one of the underlying blocks in shaping how we interact with environment and achieve tasks, the papers shows an interesting insight of how combining functional affordances, spatial proximity and conceptual similarity helps in planning and acting in human-centered environment.

The second paper titled, "Learn like Infants: A Strategy for Developmental Learning of Symbolic Skills Using Humanoid Robots", authored by Kun Li and Max Q.-H. Meng, emphasizes on the importance of symbolic representation and semantics level reasoning for intuitive and more natural interaction of the robot in human-centered environment. Inspired by underlying phases of sensorimotor object learning process from child development psychology, the paper presents an interesting strategy implemented on humanoid robot to facilitate autonomous incremental learning of objects (rigid as well as articulated) by building different layers of object models starting from sample-based to symbolic-based representation, without much need of prior knowledge.

In the third paper, "Inference of Human Beings' Emotional States from Speech in Human-Robot Interactions" the authors Laurence Devillers, Marie Tahon, Mohamed A. Sehili and Agnes Delaborde focus on one of the important blocks constituting the development of social interaction capabilities: perception of emotion in real environment. The aim is to build a dynamic representation of human emotional and interaction tendencies, so that the robot can also modulate its behavior appropriately. Using audio input, the method combines predicted output from interestingly six different systems to detect four emotions: anger, joy, sadness and neutral. Results based on the large realistic databases, also involving potential end user (elderly people), show the gain in reliability and accuracy of the detected emotions.

The fourth paper is on "Evaluating the Engagement with Social Robots", authored by Salvatore M. Anzalone, Sofiane Boucenna, Serena Ivaldi and Mohamed Chetouani. This paper proposes a set of metrics to evaluate the notion of engagement during social human robot interaction. A combination of static and dynamic attributes of social interaction development, such as attention focus, joint attention, synchrony, along with other information is used to evaluate the interaction quality in face-to-face human-robot social interaction scenarios. Two interesting case studies, interactive environment knowledge building and autism assessment have been presented, emphasizing on the importance of incorporating non-verbal aspects and attributes both in evaluation as well as in behavior design of socially interactive robots.

The fifth paper "Combining Robotic Persuasive Strategies: The Persuasive Power of a Storytelling Robot that Uses Gazing and Gestures", authored by Jaap Ham, Raymond H. Cuijpers and John-John Cabibihan, explores how the combination of basic modalities of social interaction, such as gaze and gesture, affects the persuasiveness of a socially interactive robot and how social robots can be made more persuasive if required. A storytelling context to the human by a robot is used to measure the combined and separate influences of robot's gaze and persuasive gesture. Some interesting findings, their agreements and disagreements with some earlier studies in human psychology, and their link with human robot interaction and perception of robots are discussed.

The sixth paper titled "This robot is sociable: close-up on the gestures and measured motion of a human responding to a proactive robot", authored by Ritta Baddoura and Gentiane Venture, studies an interesting scenario in which the participant were unaware of the fact that a humanoid robot might enter in the room and interact with them. The study focuses on understanding the relation between the perceived sociability of the robot with respect to the tendency to respond by the human to the robot. Patterns of arm and head motion of the human interacting with the robot have been analyzed and relations with participants' answers to questionnaire are discussed, serving as pointers about how the users' reactions and gestures can be indicator of their perception about a social robot and the success of interaction.

The seventh paper is on "Why should we imitate robots? Effect of back imitation on judgement of imitative skill", authored by Yasser Mohammad and Toyoaki Nishida. Inspired from child developmental research and evidences on the presence and role of mutual imitation process, the authors present and evaluate an interesting hypothesis on the effect of imitating a robot by humans (back imitation) on their perceptions of imitative skills of the robot. Results and discussion through three studies point about the perceptions of robot's imitative skills, human-like motion and interaction quality. The paper also discusses the links of such back imitation with the human intention of future interaction with the robot and points towards factors to be considered for humanrobot interaction and learning from demonstration designs.

The eighth paper is titled "The Design, Development, and Deployment of RoboParrot for Screening Autistic Children" and is authored by Pegah Soleimman Dehkordi, Hadi Moradi, Maryam Mahmoudi, Hamid Reza Pouretemad, Soehil Salehi-Mobarakeh and Morteza Ghavami. It investigates an interesting societal application of social robots: identifying the autism like developmental disorder in children. Three categories of robot functionalities (sound \& voice, 
body movement and touch sensing) have been combined to develop some social child-robot interactions. Based on interaction patterns of different groups of children, some basic clues of social development and distinguishing features have been identified as indicators for children with autism.

In the ninth paper, titled "The Impact of Social Robotics on L2 Learners' Anxiety and Attitude in English Vocabulary Acquisition", the authors M. Alemi, A. Meghdari and M. Ghazisaedy aim to explore application of a social robot assistant for teaching foreign languages to young students. Based on the findings that anxiety and attitude are crucial factors affecting the second language acquisition process, the paper studies the effect of robot assisted teaching. The robot was programmed with some social interaction behaviors, such as calling on students to answer, cheering the correct answer, making mistakes on purpose, gesture and dialog based acting for a word, etc. The paper outlines some interesting finding about the motivation levels of the students, their engagement in learning and their beliefs about self-progress.

The tenth paper, titled "The Effect of Embodiment in Sign Language Tutoring with Assistive Humanoid Robots", is authored by Hatice Köse, Pinar Uluer, Neziha Akalın, Rabia Yorgancl, Ahmet Özkul and Gökhan Ince. It presents an application of robot, equipped with basic capability of generating actions and feedback based on sign language, to serve as assistant in sign language tutoring for children with communication impairments. Inspired from the studies suggesting that during preoperational stage of child development, knowledge is represented by language, mental imaginary and symbolic thoughts, in the paper interactive language games (based on daily life signs) have been developed in two different embodiment conditions: physical and virtual. Interesting results of the study involving normal and hearing-impaired children have been discussed, indicating the role of physical embodiment on children's engagement and motivation.

\section{Acknowledgements}

We would like to thank the Editor in Chief Prof. Shuzhi Sam $G e$ for inviting us to organize this special issue, Dr. Hongsheng He, Mr. Anand David and Ms. Nathalie Jacobs from Springer for their efforts in bringing the special issue a reality.

We are also very much thankful to the invited reviewers: Fernando Alonso-Martín, Danilo Bruno, Sylvain Calinon, Aurélie Clodic, Joachim de Greeff, Lavindra De Silva, Ravindra Senarathna De Silva, Nicola Doering, Mauro Dragone, Victor Gonzalez-Pacheco, Horst-Michael Gross, Thomas Holz, Chien-Ming Huang, Qiang Huang, Odest Chadwicke Jenkins, David O Johnson, Alexandra Kirsch, W. Bradley Knox, Séverin Lemaignan, Angelica Lim, Manja Lohse,
Maryamossadat N. Mahani, Luis Felipe Marin-Urias, Bilge Mutlu, Mohammad Obaid, Dennis Perzanowski, Robin Read, Miguel A. Salichs, Jochen Triesch, Kadir Firat Uyanik, Gregor Wolbring, for their valuable time, input, suggestions and comments, which greatly helped the editors in decision making process and encouraged authors to elevate the quality of their papers.

We would also like to express our gratitude to Dr. Peter Ford Dominey, Mr. Rodolphe Gelin, Prof. Inaba Masayuki, Prof. Alessandro Saffiotti, Dr. Riichiro Tadakuma for their valuable supports and advices. We would also like to thank the members of Socially Intelligent Robots and Societal Applications (SIRo-SA) topic group of euRobotics, and the Romeo 2 project (Humanoid Robot Assistant and Companion for Everyday Life) and its consortium for their valuable supports and contributions in the special issue.

Without all these great support and collaborative effort, the special issue would not have taken its matured and vibrant shape towards communicating the strengths, needs, challenges and applications of applied aspects of developmental social robotics.

\section{Conclusions}

This special issue has touched an important aspect of developmental robotics: the applied side in the domain of social robotics. The special issue incorporates a balance of high quality research papers on embodiment mechanisms, evaluations, design guidelines and applications of some of the fundamental building blocks and capabilities constituting the development of social intelligence. Each of the paper also triggers interesting future directions to explore. We hope this special issue will serve to the social robotics community, further evoke cross-disciplinary and multidisciplinary scientific collaborations, enrich our understanding about potential societal applications of social robots and pave the way for further investigations, towards building harmonious relationship among humans and robots for betterment of society.

Dr. Amit Kumar Pandey is the Chief Scientist (R\&D) and scientific coordinators of collaborative projects at Aldebaran SoftBank Group, Paris, France. Earlier for 6 years he worked as doctoral and postdoctoral researcher in Robotics and AI at LAAS-CNRS (French National Center for Scientific Research), Toulouse, France. His Ph.D. thesis (Towards Socially Intelligent Robots in Human Centered Environment, 2012, INSA, Univ. of Toulouse/LAAS-CNRS) is the second prize winner (tie) of the prestigious Georges Giralt Award 2013 for the best thesis in Robotics in entire Europe, awarded by European Robotics Research Network-euRobotics. His current research interest includes Socially Intelligent Robots, Human Robot Interaction (HRI) and Robot's Cognitive Architecture. He has been actively contributing and involved in the design and development of various national and European Union (EU) projects. He is the founding coordinator of the Socially Intelligent 
Robots and Societal Applications (SIRo-SA) topic group of euRobotics, and actively contributing in the Multi-Annual Roadmap (MAR) of euRobotics, which aims to shape the future of robotics in Europe in partnership with the European Commission (EC).

Dr. Rachid Alami is Research Director at LAAS-CNRS. He received an engineering diploma in computer science in 1978 from ENSEEIHT, a Ph.D. in Robotics in 1983 from University Paul Sabatier (Toulouse, France) and a Habilitation à diriger des recherches in 1996 . He contributed and took important responsibilities in several national, European and international research and/or collaborative projects (EUREKA, ESPRIT, IST). His main research contributions fall in the fields of robot control architectures, task and motion planning, manipulation, multi-robot cooperation, human-robot interaction, and more generally robot decisional autonomy. He has also a substantial experience in robotics system integration and transfer operations.

Prof. Kazuhiko Kawamura received the B.E. degree from Waseda University, Tokyo, the M.S. degree from the University of California, Berkeley, and Ph.D. degree from the University of Michigan, Ann
Arbor, all in electrical engineering. Since 1981, he held various positions within Vanderbilt University where he currently is a research professor of electrical engineering. Professor Kawamura is an internationally known researcher and educator in the fields of intelligent robots, cognitive systems and humanoid robotics. He has published over 170 research papers, a book, and book chapters in the fields of intelligent systems, robot control architecture, cognitive control, and human-robot interactions. He directs research in cognitive systems, robot skill learning, and cognitive robot development at Vanderbilt. He was a visiting Professor of Cybernetics at the University of Reading, UK in 2004 where he conducted research on cognitive robotics and working memory. Dr. Kawamura is the Founding Chair of the Technical Committee on Service Robots for the IEEE Robotics and Automation Society and was General Chairs of three IEEE Conferences (SMC 2000, RO-MAN 2005 and Humanoids 2010). He has been Editorial board members of the International Journal of Humanoid Robotics, and International Journal of Human-Friendly Welfare Robotic Systems. 